\title{
Unknown Tumor Status
}

National Cancer Institute

\section{Source}

National Cancer Institute. Unknown Tumor Status. NCI Thesaurus. Code C158709.

The tumor status is unknown. 